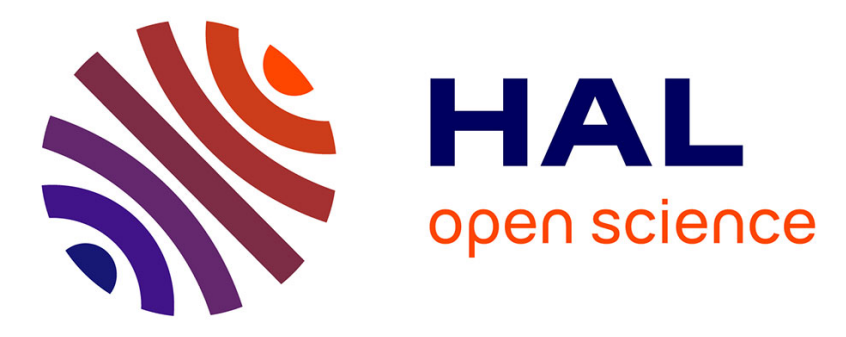

\title{
Driving Behavior Assessment and Anomaly Detection for Intelligent Vehicles
}

\author{
Chule Yang, Alessandro Renzaglia, Anshul Paigwar, Christian Laugier, \\ Danwei Wang
}

\section{- To cite this version:}

Chule Yang, Alessandro Renzaglia, Anshul Paigwar, Christian Laugier, Danwei Wang. Driving Behavior Assessment and Anomaly Detection for Intelligent Vehicles. CIS-RAM 2019 - 9th IEEE International Conference on Cybernetics and Intelligent Systems (CIS) Robotics, Automation and Mechatronics (RAM), Nov 2019, Bangkok, Thailand. pp.1-6. hal-02355809

\section{HAL Id: hal-02355809 \\ https://hal.inria.fr/hal-02355809}

Submitted on 8 Nov 2019

HAL is a multi-disciplinary open access archive for the deposit and dissemination of scientific research documents, whether they are published or not. The documents may come from teaching and research institutions in France or abroad, or from public or private research centers.
L'archive ouverte pluridisciplinaire HAL, est destinée au dépôt et à la diffusion de documents scientifiques de niveau recherche, publiés ou non, émanant des établissements d'enseignement et de recherche français ou étrangers, des laboratoires publics ou privés. 


\title{
Driving Behavior Assessment and Anomaly Detection for Intelligent Vehicles
}

\author{
Chule Yang ${ }^{1}$, Alessandro Renzaglia ${ }^{2}$, Anshul Paigwar ${ }^{2}$, Christian Laugier ${ }^{2}$, Danwei Wang ${ }^{1}$
}

\begin{abstract}
Ensuring safety of both traffic participants and passengers is an important challenge for rapidly growing autonomous vehicle technology. To this purpose, intelligent vehicles not only have to drive safe but must be able to safeguard itself from other abnormally driving vehicles and avoid potential collisions. Anomaly detection is one of the essential abilities in behavior analysis, which can be used to infer the moving intention of other vehicles and provide evidence for collision risk assessment. In this paper, we propose a behavior analysis method based on Hidden Markov Model (HMM) to assess the driving behavior of vehicles on the road and detect anomalous moments. The algorithm uses the real-time velocity and position of the surrounding vehicles provided by the Conditional Monte Carlo Dense Occupancy Tracker (CMCDOT) framework. Next, by associating with the road information, the movement of each vehicle can be classified into several observation states, namely, Approaching, Braking, Lane Changing, and Lane Keeping. Finally, by chaining these observation states using a Markov model, the abnormality of driving behavior can be inferred into Normal, Attention, and Risk. We perform experiments using CARLA simulator environment to simulate abnormal driving behaviors, and we provide results showing the successful detection of abnormal situations.
\end{abstract}

\section{INTRODUCTION}

According to the Global status report on road safety 2018 [1], traffic accidents caused approximately 1.35 million deaths each year and up to 50 million injuries. With the rapid development of robotics, data science and sensor technology, many intelligent vehicles have appeared in our daily lives, such as Uber, Tesla, Waymo, and so forth. However, even the smartest autonomous vehicles cannot guarantee road safety and can cause lethal death or injuries [2]. Among them, the inability to detect and identify risky driving behavior in time is an essential cause of accidents.

Although many works have been done to detect the abnormal scene by using surveillance videos [3], [4] or autonomous robots [5], [6], it is still challenging to detect the anomalous moments in highly dynamic traffic scenarios. Existing works on traffic scenario mainly involves vehicle trajectory prediction [7], vehicle behavior prediction (lane-change) [8], and time to collision risk assessment [9]. Few works focus on associating the anomalous driving maneuver with the behavior risk in a traffic scenario. The abnormal behavior of the vehicle can indicate the potential danger of the driver so

\footnotetext{
1 School of Electrical and Electronic Engineering, Nanyang Technological University, 639798 Singapore; e-mail: yang0438@e.ntu.edu.sg, edwwangentu.edu.sg

2 Univ. Grenoble Alpes, Inria, Chroma, F-38000 Grenoble, France; e-mail: alessandro.renzaglia@inria.fr, anshul.paigwar@inria.fr, christian.laugier@inria.fr
}

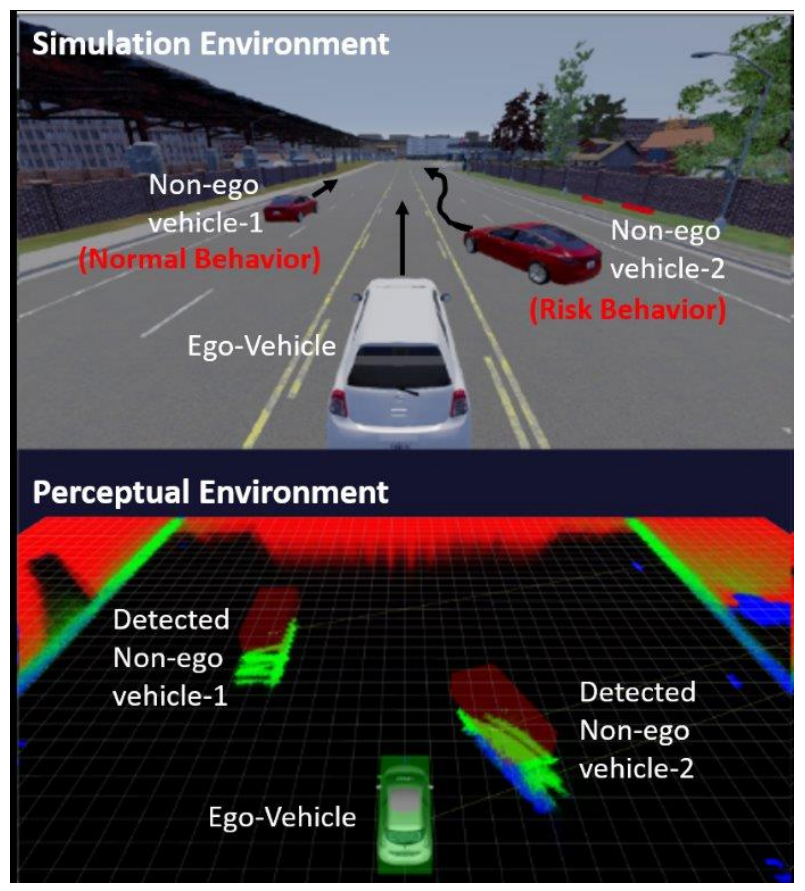

Fig. 1: (Top) Simulation environment with CARLA simulator. The white vehicle is the ego-vehicle and two non-ego vehicles are simulated to perform anomaly movements. (Bottom) Perceptual environment with CMCDOT framework. By analyzing the real-time velocity and position of the vehicle, the state and behavior of vehicles can be inferred.

that more attention should be paid to him. Thus, this paper proposes an approach to analyze the driving behavior of nonego vehicles and assess the behavior risk on the road. First, we use the Conditional Monte Carlo Dense Occupancy Tracker (CMCDOT) [10] to obtain the representative velocity and position of detected vehicles on the road. Next, by analyzing their real-time motion and position, observation states of each vehicle can be classified. The abnormality of driving behavior of each vehicle can then be inferred by chaining the observation states through Hidden Markov Model (HMM). The main contributions of this paper are as follows:

- Four observation states are defined and classified by analyzing the motion and position information obtained from CMCDOT.

- Driving behaviors are linked to observation states based on an HMM, to infer anomalous behaviors.

- We demonstrate the use of CARLA simulator [11] to generate abnormal driving behaviors and validate the 
proposed algorithm.

The rest of this paper is structured as follows. Section II reviews the related works. Section [II introduces the basic idea of our perception framework, the CMCDOT. Section IV] gives an overview of the proposed method and describe the methodology in detail. Section $\mathrm{V}$ ] shows the experimental procedures and results. Section VI concludes the paper and discusses future works.

\section{RELATED WORK}

\section{A. Trajectory Prediction}

Trajectory prediction is an essential function for autonomous vehicles to gain a more comprehensive understanding of the surrounding environments, to plan their own moving actions, and to avoid collisions. Existing methods either based on Recurrent Neural Network (RNN) or probabilistic modeling. RNN-based methods usually leverage a Long ShortTerm Memory (LSTM) network to predict a smooth trajectory for autonomous vehicles. Quite a few works study highway scenarios by applying LSTM. [12] predicts future longitudinal and lateral trajectories for vehicles by training in a large dataset in various traffic densities rather than a low number of trajectories collected from a few drivers, while [13] predicts vehicle maneuvers by learning a model that assigns probabilities for different maneuver classes, and outputs maneuver specific predictions for each maneuver class. Other research using probabilistic modeling to deal with lane-change on the road [14], and meanwhile considering the possibility of a crash with adjacent vehicles to reduce false alarms. [15] takes advantage of both physics- and maneuver-based methods and combines them to take into account both short-term and longterm effects for more accurate trajectory prediction.

\section{B. Risk Assessment}

The risk in this paper is the level of uncertainty that other vehicles introduce for ego-vehicles. For autonomous vehicles, the risk assessment needs to analyze the impact of the movement of surrounding vehicles on their normal maneuver, the likelihood of collisions, and the possibility of avoiding the danger. According to the cause of the risk, it is divided into collision risk based on future trajectory and risk based on unexpected behaviors [16]. The first type of risk assessment considers collisions to be the most critical risk and analyzes the risk based on the likelihood of collisions. The second risk assessment focuses on the dangerous situation caused by the driver's accidental operation. In the autonomous vehicle scene, the first case is more widely researched topic. [17] proposes two complementary risk indicators (collision time and minimum safety margin) in terms of time and space to cover all risk scenarios that may occur with vehicle lane changes on highways. This method reliably assesses the risk of collision. In [18], the authors present a large-scale data analysis to compare Time to Collision (TTC) and the Enhanced Time to Collision (ETTC) indicator with acceleration under a normal vehicle driving. The distribution of ETTC and TTC at the start of braking in normal following conditions is compared, and the probability model of TTC and ETTC values at braking at a series of vehicle speeds is given, which provides data for subsequent research in related fields. In [19], the three indices of the transient speed of the following vehicle, the transient speed difference between the leading and following vehicles, and the following vehicle's acceleration (FA) are used to estimate the probability of collision risk between vehicles. In fact, when these quantities are relatively large, the vehicle does not have enough time to deal with the emergency. [20] considers TTC and safety margins, as well as speed as a risk perception indicator to assess the impact of information containing risk levels. The Stop Distance Index (SDI) is obtained, and the risk level classification is performed using the collision risk index based on the SDI value.

\section{PERCEPTION FRAMEWORK: CMCDOT}

The CMCDOT [10] is a perception system able to provide a dense and generic representation of the environment [21], [22] through probabilistic occupancy grids (see Fig. 2), based on Bayesian fusion, filtering of sensor data and Bayesian inference. In particular, by tracking spatial occupancy, it infers the dynamics of the scene via a hybrid representation of the environment, which is based on static and dynamic occupancy, free spaces and unknown regions (see Fig. 3). This differentiation enables the use of state-specific models, such as classic occupancy grids for static components and sets of moving particles for dynamic occupancy, as well as confidence estimation and management of areas with no information.

Additionally, an important feature of the CMCDOT, and main subject of study of this paper, is the velocity estimation for each cell of the grid. Most of the existing velocity estimation methods consist in detecting and tracking dynamic objects in the scene. Detection of moving objects requires to predefine shape of objects and involves fitting of object models in point clouds to find the location of objects in the environment. As the number and types of objects increases, the detection and tracking becomes a difficult and costly problem to solve. The grid-based approach used in the CMCDOT framework [10] instead of detecting objects, directly computes estimations of

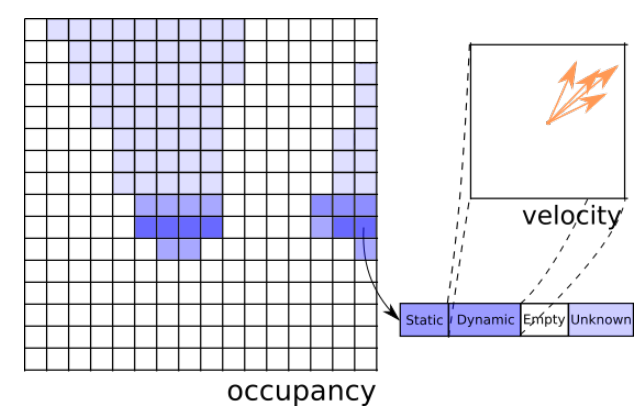

Fig. 2: Data representations in CMCDOT formulation. The environment is divided into cells, to which static, dynamic, empty and unknown coefficients are associated. The dynamic part is expressed by weighted particles which sample the velocity space. 


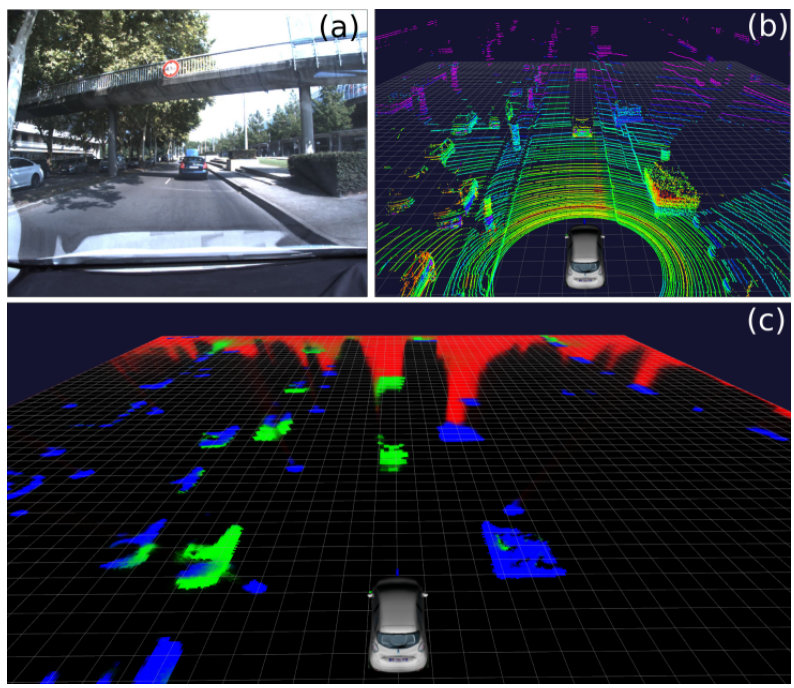

Fig. 3: (top) The environment around the vehicle as observed using a 64 layered LiDAR. (Bottom) Representation of the environment as dense probabilistic occupancy grid output from CMCDOT framework. Colors represents different states of each cell in the grid: occupied static (blue), occupied dynamic (green), empty (black), unknown area (red).

the position of every cell of the grid. By comparing these estimations with the ones obtained in previous time steps, the distinction between static and dynamic cells can be easily made, which in turn helps to estimate the velocity (speed and direction of motion) for each cell.

\section{Methodology}

The overall workflow adopted for this work is shown in Fig. 4. The CMCDOT framework takes a point cloud and odometry data as input, generates an occupancy grid map and estimates velocities for each cell. A 3D object detection algorithm [23] is then used for detecting the position $x$ of the vehicles and the bounding boxes. The representative velocity $v$ for each vehicle is obtained by taking the mean value of the cells within the bounding box of the vehicle. By tracking and analyzing the real-time motion and position between the nonego vehicle and the ego-vehicle for consecutive time-steps, observation states can then be obtained. Finally, the driving behavior of each non-ego vehicle is inferred through a HMM.

In order to validate our methodology comprehensively, we rely on CARLA simulator to generate a large number of scenarios with abnormal driving behavior by parameterizing the movements of the vehicles. Simulation setup and scenario description are explained in detail in section V.

For each vehicle $j$ detected in CMCDOT, we extract its overall velocity $v_{j}$, acceleration $a_{j}$ and position $x_{j}$. For the ego-vehicle, the perceptual parameters are denoted as $v_{e}, a_{e}$, and $x_{e}$, respectively.

\section{A. Motion and Position Analysis}

To determine the movement state (observation state) of each non-ego vehicle, we first need to track and analyze their

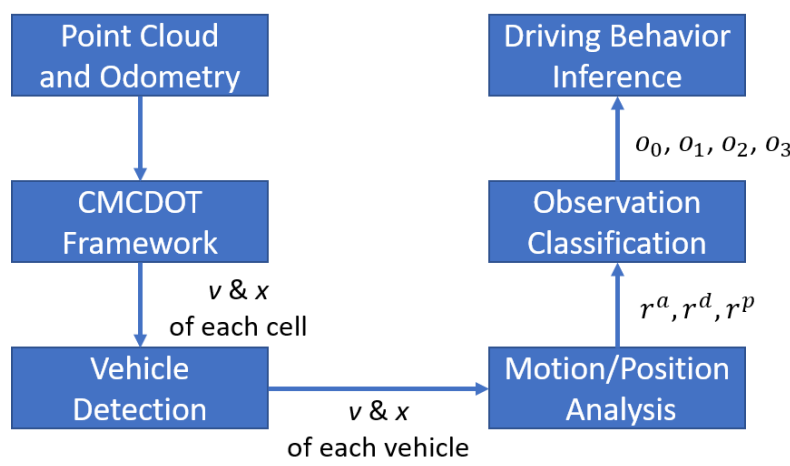

Fig. 4: Abnormal driving behavior detection pipeline.

real-time motion and position and calculate the behavioral risk according to the distance, acceleration, and direction of velocity. After that, we normalize it to $[0,1]$, and the resulting standardized behavioral risk means the degree of stability of driving behavior on the above three indicators. For each detected vehicle $j$, the corresponding risks are calculated as follows:

$$
r_{j}^{a}=P\left(r_{j}^{a} \mid a_{j}, a_{e}\right)=\frac{e^{a_{j}-a_{e}}-e^{a_{e}-a_{j}}}{e^{a_{j}-a_{e}}+e^{a_{e}-a_{j}}}, r_{j}^{a} \in[-1,1]
$$

The acceleration risk $r_{j}^{a}$ represents the relative acceleration between the vehicle $j$ and the ego-vehicle. The greater the fluctuation in speed, the larger the absolute value of the risk.

$$
r_{j}^{d}=P\left(r_{j}^{d} \mid v_{j}, l\right)=\cos \left(\frac{v_{j}}{\left|v_{j}\right|}, l\right), r_{j}^{d} \in[0,1]
$$

The direction risk $r_{j}^{d}$ indicates the degree to which the vehicle $j$ deviates from the expected road direction. $l$ is a unit vector indicating the expected moving direction of the road. The larger the risk value, the smaller the deviation from the expected direction.

$$
r_{j}^{p}=P\left(r_{j}^{p} \mid x_{j}, x_{e}, v_{j}\right)=\cos \left(\frac{v_{j}}{\left|v_{j}\right|}, \frac{x_{e}-x_{j}}{\left|x_{e}-x_{j}\right|}\right), r_{j}^{p} \in[-1,1]
$$

The position risk $r_{j}^{p}$ denotes the relative position between the vehicle $j$ and the ego-vehicle. If the risk is greater than 0 , it means that the vehicle $j$ is in front of the ego-vehicle. Otherwise, the vehicle $j$ is located behind the ego-vehicle.

\section{B. Observation Classification}

As illustrated in Fig. 5, the observation state $O$ is defined as the movement status of vehicle that can be directly observed by the ego-vehicle. In this study, the possible movements are classified into four states, namely: Approaching $\left(o_{0}\right)$, Brake $\left(o_{1}\right)$, Lane Changing $\left(o_{2}\right)$, Lane Keeping $\left(o_{3}\right), O \in$ $\left[o_{0}, o_{1}, o_{2}, o_{3}\right]$.

Let $\alpha, \beta, \gamma \in[0,1]$ define thresholds for the respective acceleration risk, direction risk, and position risk.

1) Approaching: Approaching means that the position of the detected vehicle is behind the ego-vehicle, accelerating or having a higher speed than the ego-vehicle, and the direction of movement remains almost unchanged. 


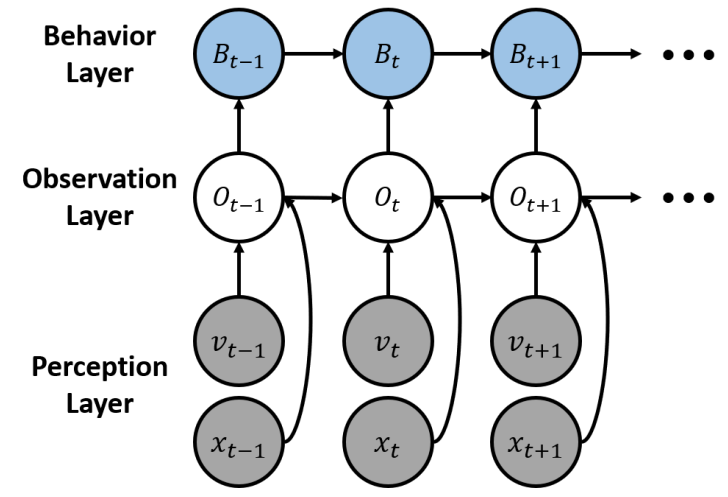

Fig. 5: Graphical model of the proposed algorithm. $v$ and $x$ are the velocity and position of each vehicle, $O$ is the observation state, and $B$ is the inferred vehicle driving behavior.

2) Brake: Brake means that the position of the detected vehicle is in front of the ego-vehicle, decelerating or having a lower speed than the ego-vehicle, and the direction of movement remains almost unchanged.

3) Lane Changing: Lane Changing means that the direction of the velocity of the detected vehicle is deviating from the expected direction (predetermined by the lane).

4) Lane Keeping: Lane Keeping means that the direction of the velocity the detected vehicle is consistent with the expected direction (predetermined by the lane).

Thus, the observation state can be obtained as follows:

$$
O=\left\{\begin{array}{l}
o_{0}, \text { if } r_{j}^{a} \geq \alpha \text { and } r_{j}^{d}>\beta \text { and } r_{j}^{p} \geq \gamma \\
o_{1}, \text { if } r_{j}^{a}<-\alpha \text { and } r_{j}^{d}>\beta \text { and } r_{j}^{p}<\gamma \\
o_{2}, \text { if } r_{j}^{d} \leq \beta \\
o_{3}, \text { otherwise }
\end{array}\right.
$$

\section{Driving Behavior Inference}

The inferred abnormality of driving behavior $B$ of the detected vehicle determines how much attention the egovehicle needs to allocate to it. In this study, the behavior can be inferred as $\operatorname{Normal}\left(b_{0}\right)$, Attention $\left(b_{1}\right)$, and $\operatorname{Risk}\left(b_{2}\right)$, $B \in\left[b_{0}, b_{1}, b_{2}\right]$. Thus, for each detected vehicle $j$, the inferred behavior at timestamp $t$ can be obtained as follows:

$$
P_{j}\left(B_{t} \mid O_{1: t-1}\right)=\sum_{B_{t-1} \in \mathcal{B}} \underbrace{P_{j}\left(B_{t} \mid B_{t-1}\right)}_{\text {Behavior Transition }} \cdot \underbrace{P_{j}\left(B_{t-1} \mid O_{1: t-1}\right)}_{\text {Previous Belief }},
$$

\section{Simulation \& Results}

To validate our approach we rely on simulated scenarios generated with the CARLA urban driving simulator [11]. This is motivated by the fact that obtaining real data for abnormal driving can be unsafe. Furthermore, our detection pipeline is only based on two sensors: LiDAR and wheel odometer. LiDARs generate point clouds by measuring the time a beam of light takes to return after hitting an obstacle in the path. In comparison to other sensors (e.g. cameras) simulated LiDAR sensor data are very similar to real data [24].

CARLA simulation environment consists of complex urban layouts, buildings, and vehicles rendered in high quality, allowing for a realistic representation of real-world scenarios. The ego-vehicle and its sensors, as well as other moving vehicles, as depicted in Fig. 3, are configured in the simulation to match with the actual system. We developed a parameterbased approach which streamlines the process through which the velocities, position and steering angle of non-ego vehicles are specified.

\section{A. Scenario Description}

Our simulation scenario focuses on the behavior of cars in a multi-lane highway. For simplification, we bound our simulation to a scenario with one ego vehicle and two nonego vehicles. Also, we fix the lanes and behavior for each simulated vehicles, as shown in Fig. 6. Non-ego vehicle-1 is in left lane moving forward with randomized acceleration and deceleration parameters over time. Non-ego vehicle- 2 is in the right lane also moving forward but with randomized minor alterations in steering angle. The ego vehicle is in the center lane moving forward with a constant velocity.

\section{B. Results}

The proposed method has been tested on the simulated scenario presented in Fig. 6 and the assessment results of the non-ego vehicle-2 are shown in Fig. 7 and Fig. 8 . In these figures, the first two charts show the real-time relative position between the ego-vehicle and the detected vehicle and their velocities. The third chart shows the observation sequence of the detected vehicle, where $o_{0}, o_{1}, o_{2}, o_{3}$ represent Approaching, Brake, Lane Changing, and Lane Keeping. The last chart shows the inferred driving behavior of the detected vehicle, where $b_{0}, b_{1}, b_{2}$ represent Normal, Attention, and Risk.

The inference result of non-ego vehicle-2 is shown in Fig. 77. In the beginning, the non-ego vehicle- 2 is located in front of the ego-vehicle but is caught and overtaken by the egovehicle within the next 20 seconds. The second chart describes the real-time velocity (both longitudinal and lateral) of the ego-vehicle and the non-ego vehicle-2. The ego-vehicle keeps a relatively steady speed in both longitudinal and lateral direction, while the non-ego vehicle-2 has a fluctuation of velocity either on magnitude or direction. The speed variation on the longitudinal direction will cause the observed state to be Approaching or Brake according to their relative position, and the speed fluctuation on the lateral direction will cause the observed steady to be Lane Changing. As a result, the behavior can be inferred as Attention when the motion of non-ego vehicle-2 is fluctuating. But if the fluctuation is beyond a certain level, it will be judged as Risk. As you can see the timestamp at around 780 and 786, the sudden speed change of the non-ego vehicle-2 leads to the observed states to be Lane Changing and Brake, which leads to the inference to be Risk.

Similar to non-ego vehicle-2, the inference result of non-ego vehicle-1 is shown in Fig. 8. The non-ego vehicle-1 keeps a similar motion and relatively stable distance to the ego-vehicle, but have some sudden changes in the direction and magnitude of velocity. The proposed algorithm successfully detect the 


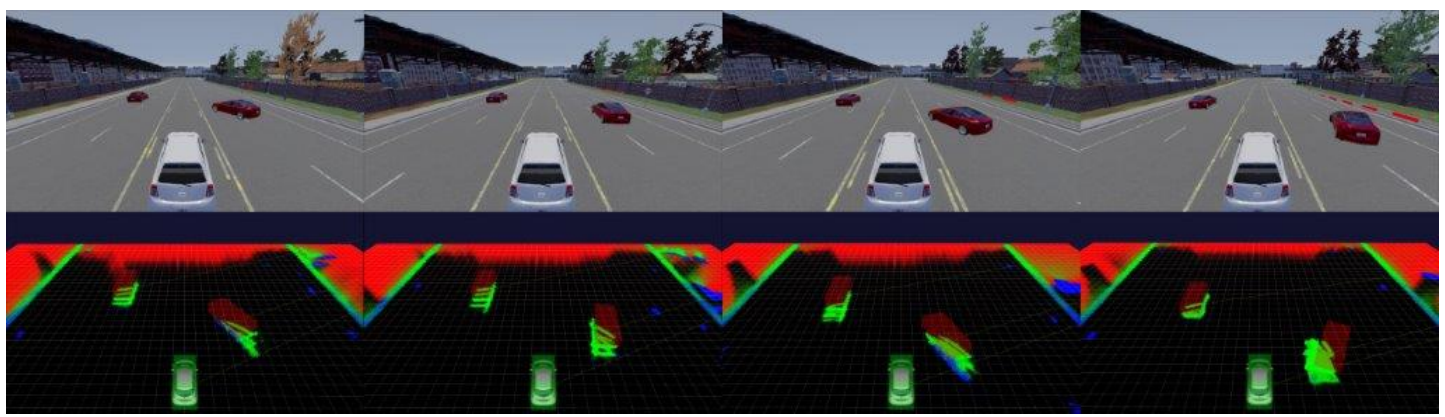

Fig. 6: Simulation environment and scenario in this study. In the upper part of this figure, the white car is the ego-vehicle, and the red car on the right is the detected vehicle with some risk behaviors. The lower part of his figure shows the perceptual results by CMCDOT.
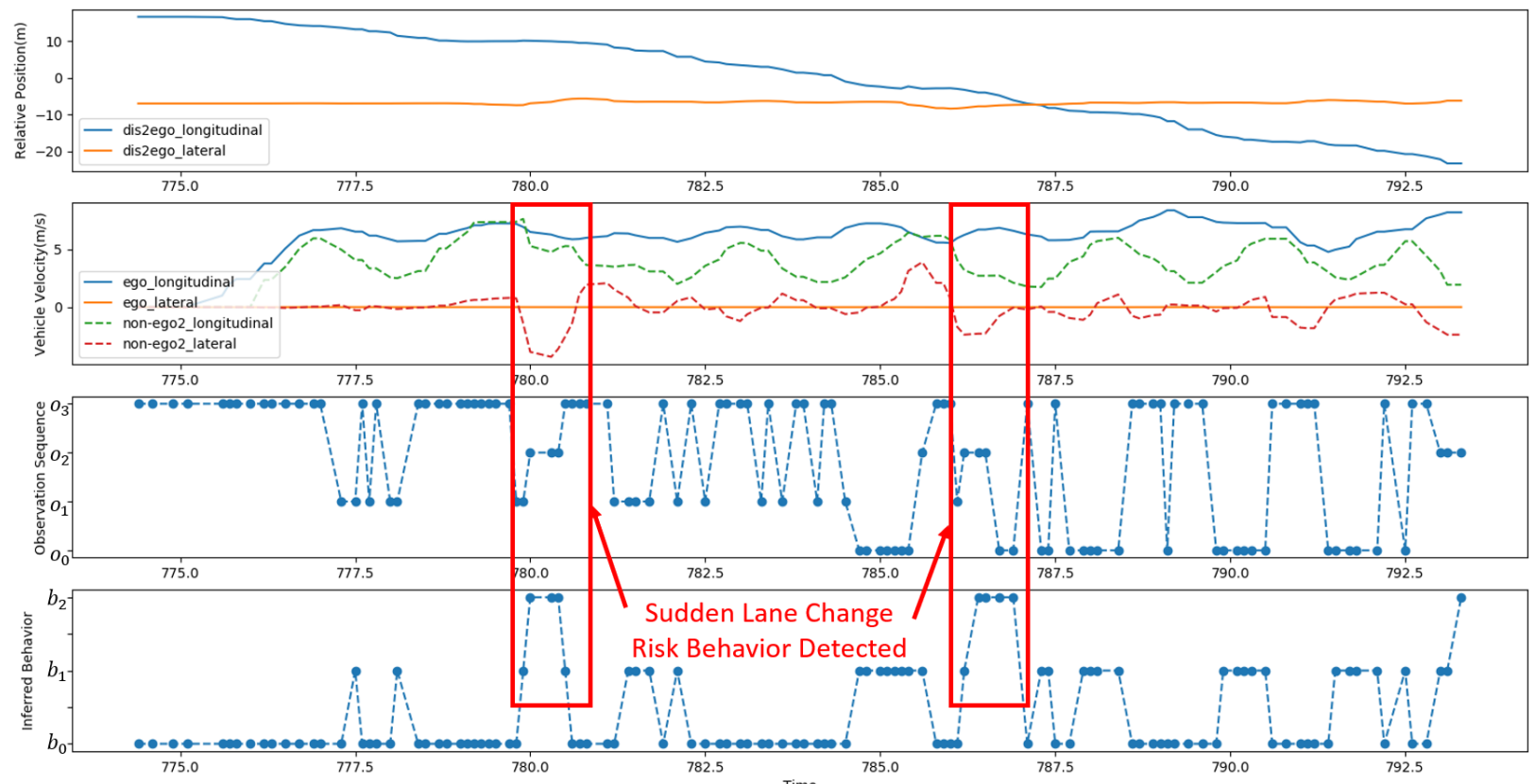

Fig. 7: Experimental results of the non-ego vehicle-2. Sudden lane changes toward the ego-vehicle are detected and anomalous moments are inferred.

sudden Brake and sudden Lane Changing, and inferred to Risk after a period of Attention.

\section{CONCLUSIONS}

In this paper, we proposed a systematic approach for vehicle driving behavior assessment and detection of anomalous moments. The proposed method takes advantage of CMCDOT framework to obtain real-time motion and velocity of vehicles. Then, the ego-vehicle can observe the movements of nearby vehicles and infer their driving behavior by tracking and analyzing their motion and position. We used CARLA simulator to generate several typical abnormal movements for two vehicles. The results show that the proposed method can successfully detect the risk moments. For future work, we intent to use machine learning methods, such as learning from demonstrations, to improve behavioral reasoning. We will also add more road users, such as pedestrians or buses, to study the impact of different semantic objects on decision making.

\section{ACKNOWLEDGMENT}

This work was supported in part by the ST EngineeringNTU Corporate Laboratory through the NRF Corporate Laboratory@University Scheme.

This work has also been conducted within the ENABLES3 project that has received funding from the ECSEL joint undertaking under grant agreement NO 692455. This joint undertaking receives support from the European Union's Horizon 2020 research and innovation programme and Austria, Denmark, Germany, Finland, Czech Republic, Italy, Spain, Portugal, Poland, Ireland, Belgium, France, Netherlands, United Kingdom, Slovakia, Norway.

\section{REFERENCES}

[1] "Global status report on road safety 2018," World Health Organization, 2018.

[2] T. B. LEE, "The hype around driverless cars came crashing down in 2018," https://arstechnica.com/cars/2018/12/uber-tesla-and-waymo-allstruggled-with-self-driving-in-2018/, 2018. 


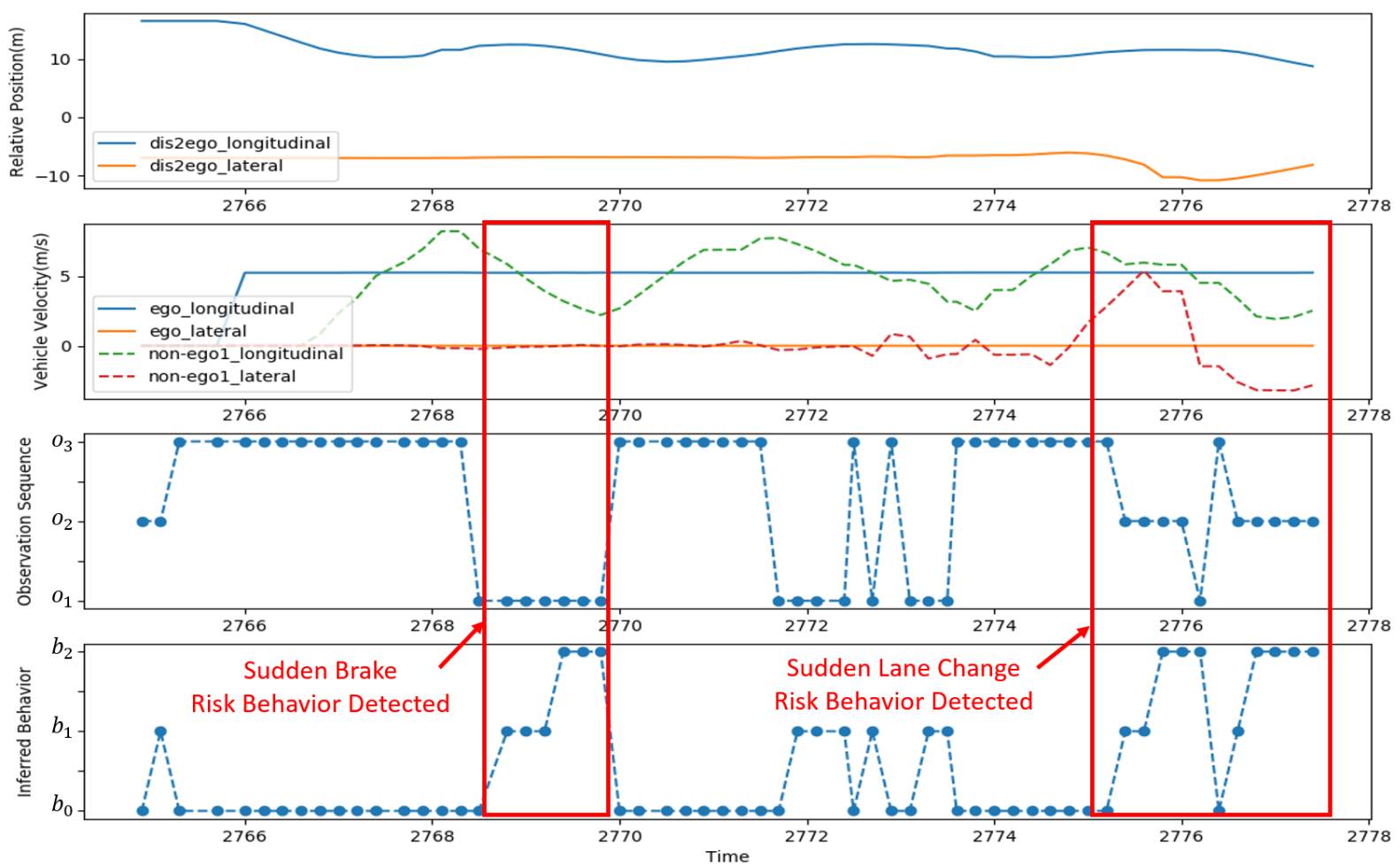

Fig. 8: Experimental results of the non-ego vehicle-1. Sudden brake and lane change toward the ego-vehicle are detected and anomalous moments are inferred.

[3] K. Lin, S.-C. Chen, C.-S. Chen, D.-T. Lin, and Y.-P. Hung, "Abandoned object detection via temporal consistency modeling and back-tracing verification for visual surveillance," IEEE Transactions on Information Forensics and Security, vol. 10, no. 7, pp. 1359-1370, 2015.

[4] Y. Lin, Y. Tong, Y. Cao, Y. Zhou, and S. Wang, "Visual-attention-based background modeling for detecting infrequently moving objects," IEEE Transactions on Circuits and Systems for Video Technology, vol. 27, no. 6, pp. 1208-1221, 2017.

[5] C. Yang, D. Wang, Y. Zeng, Y. Yue, and P. Siritanawan, "Knowledgebased multimodal information fusion for role recognition and situation assessment by using mobile robot," Information Fusion, vol. 50, pp. 126-138, 2019.

[6] C. Yang, Y. Yue, J. Zhang, M. Wen, and D. Wang, "Probabilistic reasoning for unique role recognition based on the fusion of semanticinteraction and spatio-temporal features," IEEE Transactions on Multimedia, vol. 21, no. 5, pp. 1195-1208, 2019.

[7] S. Glaser, B. Vanholme, S. Mammar, D. Gruyer, and L. Nouveliere, "Maneuver-based trajectory planning for highly autonomous vehicles on real road with traffic and driver interaction," IEEE Transactions on Intelligent Transportation Systems, vol. 11, no. 3, pp. 589-606, 2010.

[8] E. Galceran, A. G. Cunningham, R. M. Eustice, and E. Olson, "Multipolicy decision-making for autonomous driving via changepoint-based behavior prediction: Theory and experiment," Autonomous Robots, vol. 41, no. 6, pp. 1367-1382, 2017

[9] C. Laugier, "Dynamic scene understanding and upcoming collision prediction to improve autonomous driving safety: A bayesian approach,' in 2018 International Conference on Robotic Welding, Intelligence and Automation (RWIA'2018), 2018.

[10] L. Rummelhard, A. Négre, and C. Laugier, "Conditional monte carlo dense occupancy tracker," in IEEE 18th International Conference on Intelligent Transportation Systems, 2015, pp. 2485-2490.

[11] A. Dosovitskiy, G. Ros, F. Codevilla, A. Lopez, and V. Koltun, "CARLA: An open urban driving simulator," in Proceedings of the 1st Annual Conference on Robot Learning, 2017, pp. 1-16.

[12] F. Altché and A. de La Fortelle, "An lstm network for highway trajectory prediction," in 2017 IEEE 20th International Conference on Intelligent Transportation Systems (ITSC). IEEE, 2017, pp. 353-359.

[13] N. Deo and M. M. Trivedi, "Multi-modal trajectory prediction of sur- rounding vehicles with maneuver based lstms," in 2018 IEEE Intelligent Vehicles Symposium (IV). IEEE, 2018, pp. 1179-1184.

[14] H. Woo, Y. Ji, H. Kono, Y. Tamura, Y. Kuroda, T. Sugano, Y. Yamamoto, A. Yamashita, and H. Asama, "Lane-change detection based on vehicletrajectory prediction," IEEE Robotics and Automation Letters, vol. 2, no. 2, pp. 1109-1116, 2017.

[15] G. Xie, H. Gao, L. Qian, B. Huang, K. Li, and J. Wang, "Vehicle trajectory prediction by integrating physics-and maneuver-based approaches using interactive multiple models," IEEE Transactions on Industrial Electronics, vol. 65, no. 7, pp. 5999-6008, 2017.

[16] S. Lefèvre, D. Vasquez, and C. Laugier, "A survey on motion prediction and risk assessment for intelligent vehicles," ROBOMECH journal, vol. 1 , no. 1 , p. $1,2014$.

[17] S. Noh and K. An, "Risk assessment for automatic lane change maneuvers on highways," in 2017 IEEE International Conference on Robotics and Automation (ICRA). IEEE, 2017, pp. 247-254.

[18] R. Chen, R. Sherony, and H. C. Gabler, "Comparison of time to collision and enhanced time to collision at brake application during normal driving," SAE Technical Paper, Tech. Rep., 2016.

[19] C. Chen, X. Liu, H.-H. Chen, M. Li, and L. Zhao, "A rear-end collision risk evaluation and control scheme using a bayesian network model," IEEE Transactions on Intelligent Transportation Systems, no. 99, pp. 1-21, 2018

[20] M. F. Zavareh, A. R. Mamdoohi, and T. Nordfjærn, "The effects of indicating rear-end collision risk via variable message signs on traffic behaviour," Transportation research part F: traffic psychology and behaviour, vol. 46, pp. 524-536, 2017.

[21] A. Elfes, "Using occupancy grids for mobile robot perception and navigation," Computer, vol. 22, no. 6, pp. 46-57, 1989.

[22] H. Moravec, "Sensor fusion in certainty grids for mobile robots," $A I$ magazine, vol. 9, no. 2, p. 61, 1988.

[23] A. Paigwar, O. Erkent, C. Wolf, and C. Laugier, "Attentional pointnet for 3d-object detection in point clouds," in The IEEE Conference on Computer Vision and Pattern Recognition (CVPR) Workshops, 2019.

[24] J. Petit, B. Stottelaar, M. Feiri, and F. Kargl, "Remote attacks on automated vehicles sensors: Experiments on camera and lidar," Black Hat Europe, vol. 11, p. 2015, 2015. 